\title{
Prograde and retrograde atmospheric rotation of cloud-covered terrestrial planets
}

\section{Significance of astronomical parameters in the middle atmosphere ${ }^{\star}$}

\author{
M. Yamamoto ${ }^{1}$ and M. Takahashi ${ }^{2}$ \\ 1 Research Institute for Applied Mechanics, Kyushu University, 6-1 Kasugakouen, Kasuga 816-8580, Japan \\ e-mail: yamakatu@riam.kyushu-u.ac.jp \\ 2 Center for Climate System Research, University of Tokyo, 5-1-5 Kashiwanoha, Kashiwa 277-8568, Japan \\ e-mail: masaaki@ccsr.u-tokyo.ac.jp
}

Received 7 July 2008 / Accepted 19 September 2008

\section{ABSTRACT}

\begin{abstract}
Context. Cloud-covered middle-atmospheric dynamics are important in the strong prograde rotation (i.e. superrotation) observed in some terrestrial-planet atmospheres, such as Venus and Titan. In addition, the unified theory and the mechanism driving the general circulation could be extended to as yet unknown extrasolar Earth-sized habitable planet atmospheres.

Aims. We elucidate the dynamical process controlling prograde and retrograde atmospheric rotation induced by cloud heating in the middle atmosphere of terrestrial planets.

Methods. The terrestrial middle-atmospheric circulation is examined using a general circulation model in which astronomical parameters are varied.

Results. Middle-atmospheric circulation is strongly controlled by seasonal variation of the meridional circulation. Prograde and retrograde atmospheric rotation is determined by the direction of vertical angular momentum transport due to the meridional circulation resulting from tilting of the planetary rotation axis. The retrograde rotation rate is limited to $-\Omega$ for slowly rotating planets (where $\Omega$ is the planetary rotation rate).
\end{abstract}

Key words. hydrodynamics - Earth - planets and satellites: individual: Titan, Venus

\section{Introduction}

Meteorology and climate dynamics on Earth are useful in understanding atmospheric dynamics of planetary and astronomical objects. Theories explaining the general circulation in the Earth's atmosphere have been extended to other planetary atmospheres. Golitsyn (1970) proposed a similarity theory for planetary atmospheres, and Matsuda (1980) found characteristic patterns of the terrestrial general circulation and their multiple equilibrium states. Since 1980, parametric experiments using terrestrial general circulation models (GCMs) have been performed by several groups (e.g. Williams \& Holloway Jr. 1982; Del Genio \& Suozzo 1987; Williams 1988; Del Genio et al. 1993; Abe et al. 2005). However, the dynamics in the terrestrial middle atmosphere, which is located above the troposphere and below the thermosphere, have not been studied with a parametric approach. Recently, extrasolar planets with 5 to 10 terrestrial masses (e.g. Udry et al. 2007) have been detected with the aid of technological advances in astronomical observations. These advances are expected to reveal extrasolar Earth-sized planets. As yet, neither a unified theory nor a mechanism driving this general circulation has been established, although the need for such models is increasing with the ongoing accumulation of observational data.

\footnotetext{
* Appendix is only available in electronic form at http://www . aanda.org
}

Venus is a known terrestrial planet and is similar in size to Earth; however, its atmospheric circulation and radiative forcing are significantly different from those of Earth's atmosphere. On Venus, clouds of sulphuric acid cover the planet at altitudes between 47 and $70 \mathrm{~km}$, and a large prograde zonal flow of about $100 \mathrm{~m} \mathrm{~s}^{-1}$ is observed at the top of the cloud layer (at $65-70 \mathrm{~km}$ ) (e.g. Rossow et al. 1990). The radiative heating in optically thick clouds forces the middle-atmospheric circulation and drives strong prograde rotation (i.e. superrotation), at a rate 60 times faster than the planetary rotation rate (243 days). Optically thick clouds and haze are invariably seen on both Venus and Titan; they are conceptually similar to the extreme aerosol and dust layers that occur after extreme natural and human-induced disasters on Earth (e.g. asteroid impacts, supervolcano eruptions, and nuclear explosions). The dynamics of the general circulation induced by diabatic cloud heating in the middle atmosphere are of great importance in understanding both climate change caused by extreme natural and human-induced disasters, and the meteorology of terrestrial planets.

In a Venus-like terrestrial GCM (Del Genio et al. 1993) under the condition that the solar heating maximum is located above the cloud layer (in which the atmosphere is stable) far from the surface (near which the equatorial atmosphere is unstable), superrotation develops in the upper region of the model atmosphere, but not in the lower region. In our recent GCM (Yamamoto \& Takahashi 2006), the nontrivial lower-atmospheric heating $\left(0.5 \mathrm{~K} \mathrm{day}^{-1}\right.$ near the surface at the 
Table 1. Experimental conditions.

\begin{tabular}{ccccc}
\hline \hline Exp. & $T_{\Omega}($ day $)$ & $T_{\text {solar }}($ day $)$ & $T_{\text {orbit }}($ day $)$ & $-\Omega a\left(\mathrm{~m} \mathrm{~s}^{-1}\right)$ \\
\hline $\mathrm{V}$ & 243 & 117 & 225 & -1.81 \\
$\mathrm{~T}$ & 16 & 16 & 225 & -27.5 \\
$\mathrm{TS}$ & 16 & 16 & 16 & -27.5 \\
$\mathrm{E}$ & 1 & 1 & 225 & -440. \\
$\mathrm{ES}$ & 1 & 1 & 1 & -440. \\
\hline
\end{tabular}

V, T, TS, E and ES are Venus, Titan, Titan synchronous, Earth, and Earth synchronous conditions, respectively. $\Omega$ is the planetary rotation rate, $T_{\Omega}$ is the planetary rotation period, $T_{\text {solar }}$ is the solar day, $T_{\text {orbit }}$ is the orbital period, and $a$ is the planetary radius.

subsolar point) and the equator-pole surface temperature contrast $(5-10 \mathrm{~K})$ lead to a single Hadley cell, which efficiently pumps up the angular momentum in the lower and middle atmospheres. Accordingly, realistic superrotation can be reproduced, though the lower-atmospheric heating is greater than the real Venusian one.

A number of research groups (Yamamoto \& Takahashi 2003, 2004, 2006; Lee et al. 2005; Hollingsworth et al. 2007) have simulated superrotation using GCMs under Venus conditions (i.e. a surface pressure of 92 atm and a planetary rotation period of 243 days). Their results support Gierasch's mechanism (Gierasch 1975; Matsuda 1980), in which the meridional circulation efficiently pumps up the angular momentum from the lower atmosphere with the help of a large equatorward eddymomentum flux. In the present study, a GCM is extended to the middle atmosphere in cloud-covered terrestrial planets and we discuss the significance of the astronomical parameters. Although parametric GCM experiments have been conducted in previous studies (Yamamoto \& Takahashi 2006, 2007; Hollingsworth et al. 2007), their parametric ranges are too small to elucidate universal characteristics of the dynamics. In this work, we investigate the results of a large number of simulations (46 cases).

\section{Model}

In the present study, a primitive equation system (e.g. Holton 2004), in which hydrostatic equilibrium is assumed, is applied to terrestrial planetary atmospheres in the same way as Earth's climate models. Our model is established in the CCSR/NIES (Center for Climate System Research/National Institute for Environmental Study) GCM version 5.6 (Numaguti et al. 1995) using atmospheric parameters similar to those on Venus; it is utilized as a terrestrial GCM of a planet globally covered with clouds. We use simplified radiative processes that are implemented by applying 3D solar heating in the cloud layer and Newtonian cooling (see the Appendix in the online version and Yamamoto \& Takahashi 2006). The model-heating rate at the subsolar point is $30 \mathrm{~K} \mathrm{day}^{-1}$ at an altitude of $65 \mathrm{~km}$ and $0.5 \mathrm{~K} \mathrm{day}^{-1}$ near the surface. Since the heating distribution depends on the zenith angle, it is strongly related to the oblique angle of the planetary rotation axis. The equator-pole contrast of the surface potential temperature is set at $10 \mathrm{~K}$ (Joshi \& Young 2002; Yamamoto \& Takahashi 2004, 2006). The experimental conditions (the planetary rotation period $T_{\Omega}$, the solar day $T_{\text {solar }}$, the orbital period $T_{\text {orbit }}$ ) are summarized in Table 1 . The time integration starts from a motionless state and continues until the atmosphere reaches a near-equilibrium configuration.
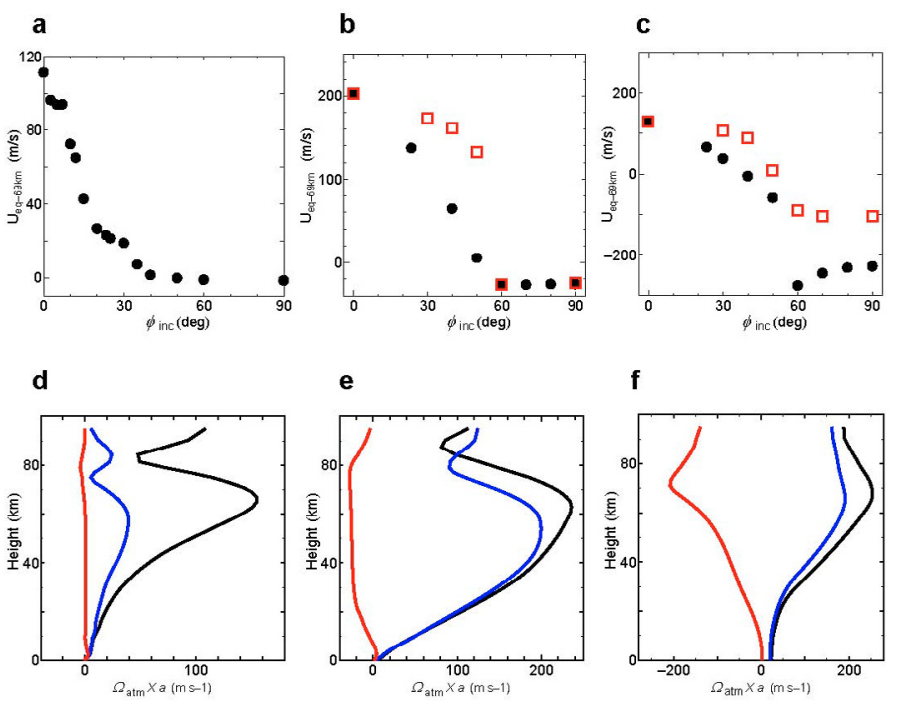

Fig. 1. Effect of the obliquity angle on the equatorial cloud-top zonal flow for a) exp. V (circles), b) exps. T (circles) and TS (squares), and c) exps. E (circles) and ES (squares). Vertical profiles of mean global atmospheric rotation rates for $\phi_{\mathrm{INC}}=0^{\circ}$ (black), 23.45 ${ }^{\circ}$ (blue), and $60^{\circ}$ (red) in exps. V d), T e), and $\mathrm{E} \mathbf{f}$ ).

\section{Results}

The upper panels of Fig. 1 show the sensitivity of the cloudtop equatorial zonal flow to the obliquity of the planetary rotation axis. For an obliquity angle, $\phi_{\mathrm{INC}}$, of zero, strong prograde atmospheric rotation is maintained. The cloud-top equatorial zonal winds decrease with increasing $\phi_{\text {INC }}$ for low and intermediate obliquity $\left(\phi_{\mathrm{INC}}=0-60^{\circ}\right)$. The wind speed is insensitive to $\phi_{\text {INC }}$ for oblique angles greater than $60^{\circ}$ in all cases. In experiments TS and E (see Table 1), we find the regime change of the atmospheric rotation state between $\phi_{\mathrm{INC}}=50$ and $60^{\circ}$. For $\phi_{\mathrm{INC}}=30-50^{\circ}$ in exps. T, TS, E, and ES, the superrotation on planets with an orbital period equal to a solar day (red squares in Fig. 1) is stronger than that on planets with a Venusian orbital period (black circles in Fig. 1). Under Earth-like conditions, the retrograde zonal flow at $\phi_{\mathrm{INC}} \geq 60^{\circ}$ is weak for short orbital periods (1 day, exp. ES) compared with that for long periods (225 days, exp. E).

For large oblique angles, $\phi_{\mathrm{INC}} \geq 60^{\circ}$ and for slowly rotating planets with 243-day and 16-day periods (exps. V, T, and TS in Figs. 1a and b), the zonal flows asymptotically approach $-\Omega a$ (where $a$ is the planetary radius), which corresponds to a motionless state with respect to the inertial frame. For rapidly rotating planets (exp. E in Fig. 1c), a strong retrograde flow is observed for large oblique angles, $\phi_{\mathrm{INC}} \geq 60^{\circ}$. However, the high wind speed is much lower than the considerably larger retrograde rotation limit $\left(-\Omega a=-440 \mathrm{~m} \mathrm{~s}^{-1}\right)$. In previously explored Earthlike GCMs (Williams \& Holloway Jr. 1982; Williams 1988), strong retrograde zonal flows were seen in the upper layer for high-obliquity experiments. The reasons for the occurrence of these strong retrograde winds will now be examined.

As mentioned above, the obliquity determines whether the atmospheric rotation is prograde or retrograde with respect to the planetary rotation. In Figs. 1d-f, the fully developed prograde atmospheric rotation is formed around the heating maximum (at an altitude of $\sim 65 \mathrm{~km}$ ) for $\phi_{\mathrm{INC}}=0^{\circ}$ and $23.45^{\circ}$, whilst retrograde rotation develops for $\phi_{\mathrm{INC}}=60^{\circ}$. For high obliquity, $\Omega_{\mathrm{atm}} a$ (where $\Omega_{\mathrm{atm}}$ is the annual and global mean atmospheric rotation rate) reaches the retrograde rotation limit, $-\Omega a$, near the 
a Exp. V

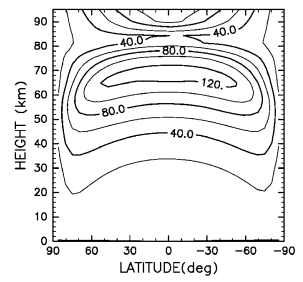

CONTOUR INTERVAL $=2.000 E+0$

b Exp. V

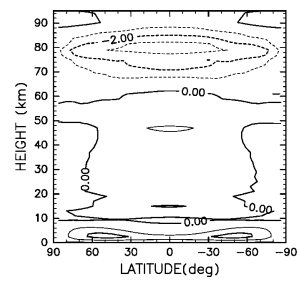

CONTOUR INTERVAL $=1.000 E+00$
Exp. T

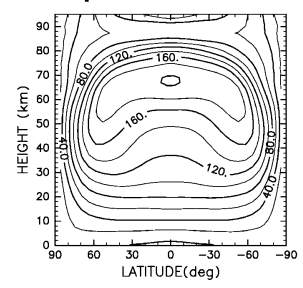

CONTOUR INTERVAL $=2.000 E+01$

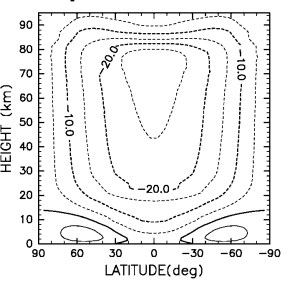

CONTOUR INTERVAL $=5.000 E+00$

$$
\text { Exp. T }
$$

Exp. E

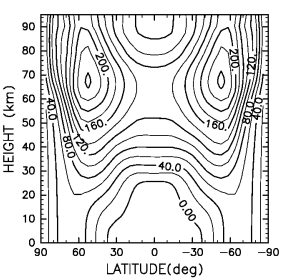

Exp. E

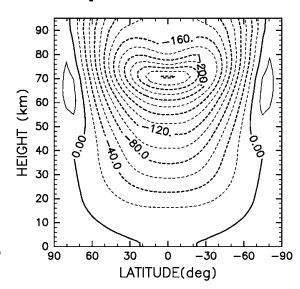

CONTOUR INTERYAL $=2.000 E+0$
CONTOUR INTERVAL $=2.000 E+$

Fig. 2. Latitude-altitude distributions of annually averaged zonal mean flow for a) $\phi_{\mathrm{INC}}=0^{\circ}$ and $\left.\mathbf{b}\right) \phi_{\mathrm{INC}}=60^{\circ}$ in exps. $\mathrm{V}, \mathrm{T}$ and $\mathrm{E}$.

cloud top, and in the broad altitude range of 40-60 km, in exps. $\mathrm{V}$ and $\mathrm{T}$, respectively. Around the retrograde rotation limit of zero atmospheric rotation in the inertial frame (i.e. $\Omega_{\mathrm{atm}}=-\Omega$ in the rotation frame), the vertical angular-momentum flux due to the meridional circulation becomes zero because of the absence of atmospheric angular momentum. This means that vertical angular momentum transport stops in the region of the retrograde rotation limit and atmospheric rotation cannot be developed, even if meridional circulation is dominant. Thus, the retrograde flow is strongly limited to $-\Omega a$ for slowly rotating planets. For rapidly rotating planets, however, the retrograde flow does not reach $-\Omega a$ because of the extremely large $\Omega$, although large retrograde velocities occur, reaching well in excess of $200 \mathrm{~m} \mathrm{~s}^{-1}$.

Figure 2 shows the latitude-height distributions of annually averaged zonal mean flows. For zero obliquity angle (Fig. 2a), the fully-developed superrotations of $>100 \mathrm{~m} \mathrm{~s}^{-1}$ are maintained in these terrestrial middle atmospheres. In slowly rotating planets (exps. V and T), the maxima of cloud-top zonal flows are located at the equator. The lower-atmospheric rotation in exp. $\mathrm{T}$ is faster than that in exp. V. In a rapidly rotating planet (exp. E), the strong mid-latitude jet of $>200 \mathrm{~m} \mathrm{~s}^{-1}$ is formed above $55 \mathrm{~km}$. For a large obliquity angle $\left(60^{\circ}\right)$, retrograde rotations are predominant in the middle atmosphere. The weak superrotations are located near the surface for exps. V and T, and in the polar regions for exp. E. The retrograde flows with nearly rigid-body rotations become larger with increasing $\Omega$.

Dynamics of the fully developed superrotation in exp. $\mathrm{V}$ is similar to those in exp. T, as shown in Fig. 3. The single Hadley cell is predominant in the whole atmosphere and the equatorward eddy angular momentum transport is found in the two cases. These characteristics support the Gierasch mechanism, in which the meridional circulation efficiently pumps up the angular momentum with the help of large equatorward eddy momentum flux. Since the effect of the obliquity on the atmospheric dynamics of slowly rotating planets such as Venus has already been discussed in Yamamoto \& Takahashi (2007), we focus on the dynamics in the rapidly rotating planets simulated in exp. E.

For $\phi_{\mathrm{INC}}=0.0^{\circ}$ in exp. E (Fig. 2a), the mid-latitude jets of $>200 \mathrm{~m} \mathrm{~s}^{-1}$ and the equatorial flow of $>140 \mathrm{~m} \mathrm{~s}^{-1}$ are located at the cloud top. Equatorial superrotation was also simulated in Earth-like two-layer GCMs (Suarez \& Duffy 1992). Angular momentum is supplied by the retrograde surface flow $\left(<0 \mathrm{~m} \mathrm{~s}^{-1}\right)$ a Exp. V

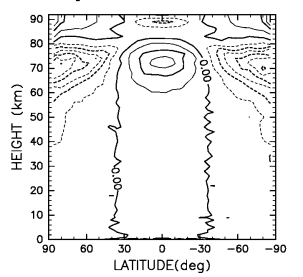

CONTOUR INTERVLL $-2.500 E-03$

b Exp. V

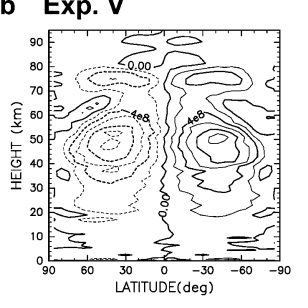

CONTOUR INTERVAL $=2.000 E+08$
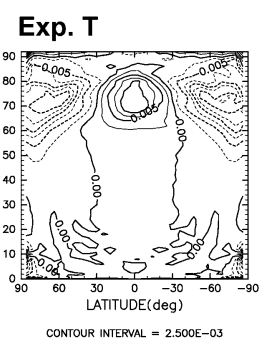

Exp. T

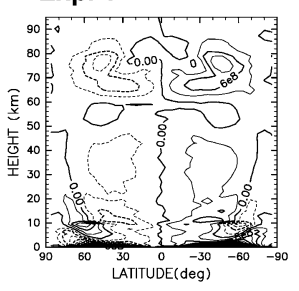

CONTOUR INTERVAL $=3.000 E+08$

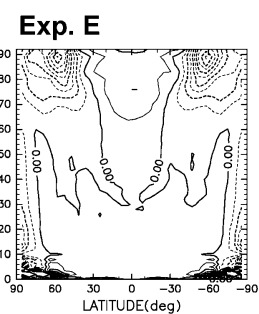

CONTOUR INTERYLL $=5.000 E-05$

Exp. E

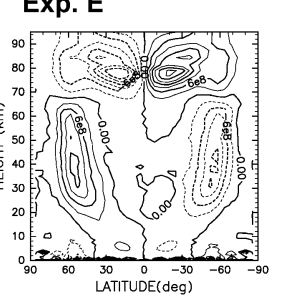

CONTOUR INTERYAL $=3.000 E+08$
Fig. 3. Latitude-altitude distributions of annually averaged a) residual mean vertical flow and $\mathbf{b}$ ) horizontal eddy angular-momentum fluxes for $\phi_{\mathrm{INC}}=0^{\circ}$ in exps. V, T and E.

through surface-friction drag. The mean global vertical angularmomentum fluxes caused by meridional circulation move upward, whereas those due to eddies move downward. The horizontal eddy-momentum flux is equatorward above the cloud top. Thus, angular momentum is transported toward the equatorial regions, where the upward flow is located. Meridional circulation efficiently pumps up angular momentum with the help of the large equatorward eddy-momentum fluxes. Even as the planet rotates rapidly (i.e. unlike both Venus and Titan), the Gierasch mechanism works above the cloud layer. Below $70 \mathrm{~km}$, the mean downward flow is confined to the polar side of the mid-latitude jet, and the horizontal eddy-momentum flux is poleward in the vertical shear region of the jets. The strong prograde atmospheric rotation is maintained by the upward angular momentum transport driven by the meridional circulation.

The equatorial and mid-latitude winds for $\phi_{\mathrm{INC}}=23.45^{\circ}$ (Earth's obliquity angle) are weaker than those for $\phi_{\mathrm{INC}}=0.0^{\circ}$ in exp. E. The equatorial jet has a high speed of $90 \mathrm{~m} \mathrm{~s}^{-1}$ at an altitude of $60 \mathrm{~km}$, compared with the Earth's middle atmosphere. Prograde winds (westerly on Earth) are dominant above $35 \mathrm{~km}$, while retrograde winds (easterly on Earth) are absent (not shown). Although a large prograde flow may be enhanced in this model by the absence of middle-atmosphere wave braking due to topographically forced as well as moist, thermally induced waves, the formation of superrotation suggests that the Earth's superrotation may have been induced by strong middleatmospheric heating in thick aerosol and dust layers resulting from extreme natural and human-induced disasters.

For $\phi_{\mathrm{INC}}=60.0^{\circ}$ (Fig. 4) in exp. E, strong retrograde atmospheric rotation is dominant throughout the year. The equatorial retrograde-jet maximum of $-200 \mathrm{~m} \mathrm{~s}^{-1}$ is slightly shifted at low latitudes, within $\pm 40^{\circ}$, although the seasonal heating amplitude is large. Below $70 \mathrm{~km}$, the mean vertical flow is upward (downward) in the summer (winter) hemisphere at the solstice. The mean global angular-momentum flux due to the meridional circulation is downward. At the equinox the heating maximum passes rapidly through low latitudes, as the magnitude of seasonal variation in diabatic heating is large. Thus, at the equinox the meridional circulation (which is symmetrical with respect to the equator) is weak near $65 \mathrm{~km}$ (Fig. 4e), and its upward angular-momentum transport is ineffective. Strong 

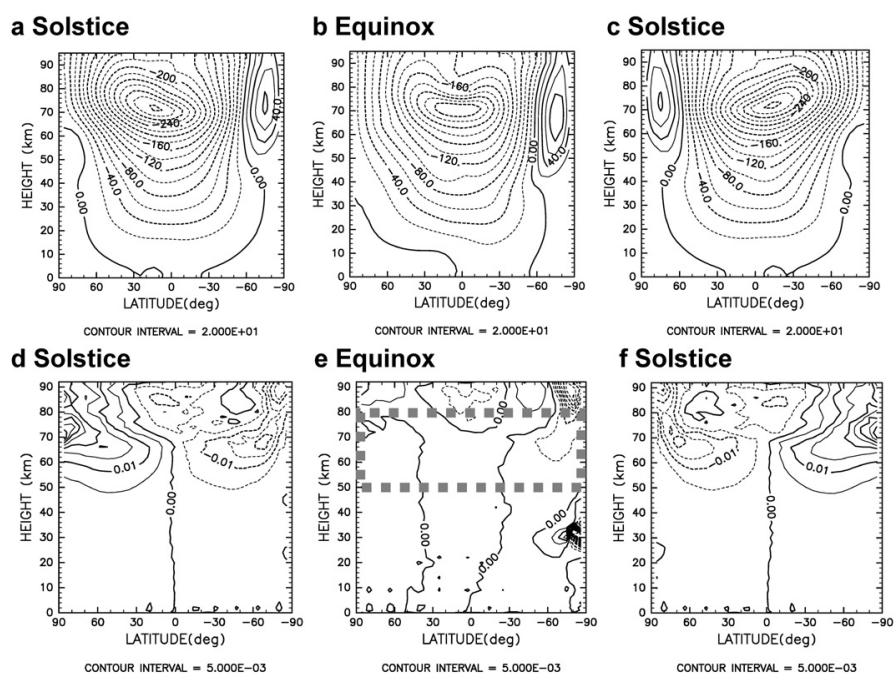

Fig. 4. Variations in seasonally averaged a)-c) zonal mean flow and df) residual mean vertical flow for $\phi_{\mathrm{INC}}=60^{\circ}$ in exp. E. The gray dotted rectangle in e) shows the area in which the meridional circulation stops at the equinox.

a

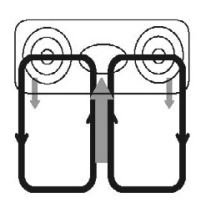

b

Solstice
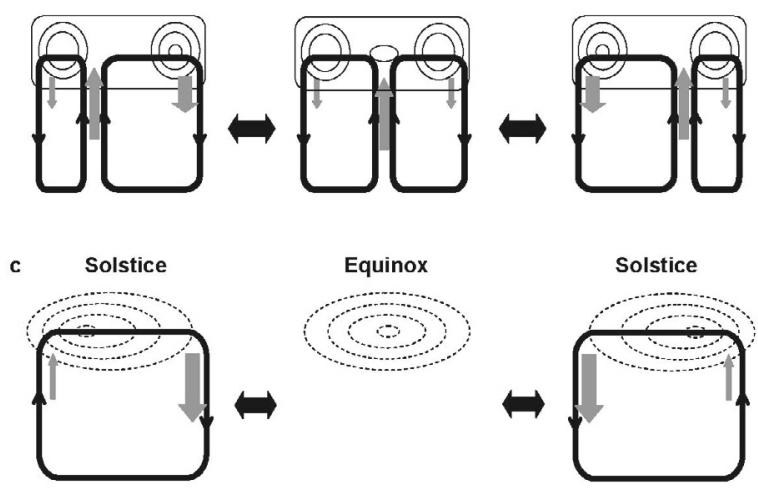

Fig. 5. Seasonal variations in the angular-momentum transport (gray arrows) caused by meridional circulation (thick black lines) for a) $\phi_{\mathrm{INC}}=0^{\circ}$, b) $\phi_{\mathrm{INC}}=23.45^{\circ}$ and c) $\phi_{\mathrm{INC}}=60^{\circ}$ in exp. E. Thin solid and dashed lines are contours of the prograde and retrograde wind speeds, respectively.

retrograde winds are maintained because the mean annual angular-momentum fluxes due to meridional circulation are downward and those due to eddies are upward at the solstice.

\section{Concluding remarks}

In the middle atmospheres of cloud-covered terrestrial planets, the prograde wind speed decreases with increasing $\phi_{\mathrm{INC}}$ for the wide parameter range. Strong superrotation is maintained in thick aerosol and haze layers on Venus and Titan because $\phi_{\text {INC }}$ is small. The regime changes of the atmospheric-rotation state can also be found in exps. TS and E. In the case of nonobliquity (Fig. 5a), superrotation in the middle atmosphere is maintained by the meridional circulation for both slowly and rapidly rotating planets. As $\phi_{\mathrm{INC}}$ increases, vertical angular momentum transport due to the meridional circulation becomes inefficient (Fig. 5b), as the meridional circulation is asymmetric with respect to the equator at the solstice; thus, the superrotation is weakened. For extremely high obliquity (Fig. 5c), since the equatorially symmetric meridional circulation is not formed at the equinox, its upward angular momentum transport is ineffective near the cloud top. In addition, downward angular momentum transport due to the meridional circulation is dominant around the solstice. Thus, a retrograde wind is formed for high-obliquity cloud-covered terrestrial planets. The condition of $\bar{u} \sim-\Omega a \cos \phi$ results in the null vertical angular momentum flux of $(\bar{u}+\Omega a \cos \phi) a \cos \phi \bar{w} \sim 0$, where $\bar{u}$ and $\bar{w}$ indicate the longitudinally averaged zonal and vertical velocities. Since the globally and annually averaged vertical angular momentum transport due to the meridional circulation stops in the middle atmosphere in the situation of $\Omega_{\text {atm }}(\equiv \bar{u} / a / \cos \phi) \sim-\Omega$, the retrograde flow cannot be further developed. Its retrograde rotation rate is limited to $-\Omega$ under the condition that the globally and annually averaged eddy angular momentum transport or frictional drag is not predominant. Thus, the retrograde rotation limit of $-\Omega$ is a significant physical quantity in the dynamics of terrestrial middle atmosphere. These new findings should be added to the general-circulation theory for terrestrial and as yet unknown extrasolar Earth-sized habitable planets.

Acknowledgements. This study was supported by a cooperative research project of the Center for Climate System Research at the University of Tokyo, and by a JSPS Grant-in-Aid for Young Scientists (B) (Nos. 17740313 and 20740273). The numerical experiments were performed on the HITACHI SR8000 and SR11000 at the Information Technology Center of the University of Tokyo and the Research Institute for Information Technology of Kyushu University. The GFD-DENNOU library was used to produce the figures.

\section{References}

Abe, Y., Numaguti, A., Komatsu, G., et al. 2005, Icarus, 178, 27 Del Genio, A. D., \& Suozzo, R. J. 1987, J. Atmos. Sci., 44, 973 Del Genio, A. D., Zhou, W., \& Eichler, T. P. 1993, Icarus, 101, 1 Gierasch, P. J. 1975, J. Atmos. Sci., 32, 1038

Golitsyn, G. S. 1970, Icarus, 13, 1

Hollingsworth, J. L., Young, R. E., Schubert, G., et al. 2007, Geophys. Res. Lett., 34

Holton, J. R. 2004, An introduction to dynamic meteorology, 4th edn. (Academic Press), 535

Joshi, M., \& Young, R. 2002, Geophys. Res. Lett., 29

Lee, C., Lewis, S. R., \& Read, P. L. 2005, Adv. Space Res., 36, 2142

Matsuda, Y. 1980, J. Meteor. Soc. Japan, 58, 443

Numaguti, A., Sugata, A., Takahashi, M., et al. 1997, CGER's Supercomputer monograph report 3, Nat. Ins. Environ. Studies, Japan, 91

Rossow, W. B., Del Genio, A. D., \& Eichler, T. 1990, J. Atmos. Sci., 47, 2053

Suarez, M. J., \& Duffy, D. G. 1992, J. Atmos. Sci., 49, 1541

Udry, S., Bonfils, X., Delfosse X., et al. 2007, A\&A, 467, L43

Williams, G. P. 1988, Climate Dyn., 3, 45

Williams, G. P., \& Holloway Jr., J. L. 1982, Nature, 297, 295

Yamamoto, M., \& Takahashi, M. 2003, J. Atmos. Sci., 60, 561

Yamamoto, M., \& Takahashi, M. 2004, Geophys. Res. Lett., 31

Yamamoto, M., \& Takahashi, M. 2006, J. Atmos. Sci., 63, 3296

Yamamoto, M., \& Takahashi, M. 2007, Geophys. Res. Lett., 34, 16202 


\section{Appendix}

The method and physical parameters used are given in Yamamoto \& Takahashi (2006). The radiative, surface, and subgrid processes in our GCM are summarized here. The heating rate of Fig. 1a in Yamamoto \& Takahashi (2006) is used as the solar heating. The 3D solar heating rate has the zenith-angle $(\lambda)$ dependence of $\cos ^{1.4} \lambda$. The infrared cooling rate $\left(\mathrm{K} \mathrm{day}^{-1}\right)$ is written as

$Q_{0}+\left(T-T_{0}\right) / \tau_{\mathrm{N}}$,

where $T$ is temperature, $T_{0}$ is reference temperature of Fig. 2 in Yamamoto \& Takahashi (2003), $\tau_{\mathrm{N}}$ is a time constant of Newtonian cooling of Fig. 1b in Yamamoto \& Takahashi (2006), $Q_{0}$ is the global mean infrared heating rate when $T=T_{0}$, which equals the global mean solar heating rate. In the calculation of the temperature, the convective adjustment is applied each time step.

Horizontal flow is dissipated by the Rayleigh friction of 30 days near the top layer. The coefficient $\alpha_{\mathrm{R}}$ is defined as

$\alpha_{\mathrm{R}}=\frac{1}{30 \text { days }}\left[1+\tanh \left(\frac{z-z_{\mathrm{T}}}{6 \mathrm{~km}}\right)\right]$,

where $z=-(5 \mathrm{~km}) \ln \sigma$ and $z_{\mathrm{T}}=-(5 \mathrm{~km}) \ln \sigma_{\text {top }}, \sigma_{\text {top }}$ denotes the sigma level of the top layer. In addition, eddy components of horizontal flow are dissipated with the same time-constant as the Newtonian cooling one in the model atmosphere. In the lowermost layer, the surface frictional drags are incorporated as

$\partial u / \partial t=-u / \tau_{\text {drag }}$

$\partial v / \partial t=-v / \tau_{\text {drag }}$

$\partial \Theta / \partial t=-\left(\Theta-\Theta_{0}\right) / \tau_{\mathrm{drag}}$,

where $\Theta$ is potential temperature. $\Theta_{0}$ is a zonally uniform reference surface potential temperature and has a latitude dependence of $\cos ^{1.4} \phi$. For the standard surface pressure of 92 atm, $\Theta_{0}$ is $732.7 \mathrm{~K}$ at the equator and $722.7 \mathrm{~K}$ at the pole. The surface drag coefficient $\tau_{\text {drag }}$ has the time constant of 3 days in this model.

The planetary boundary layer scheme included in the CCSR/NIES GCM is not used in the simplified model. The 4th order horizontal diffusion of the e-folding time of 4 days at the maximum wavenumber and the vertical diffusion of $K_{Z}=$ $0.15 \mathrm{~m}^{2} \mathrm{~s}^{-1}$ are applied in order to prevent numerical instability ( $K_{Z}$ is the vertical eddy diffusion coefficient). At the top and bottom boundaries, the vertical atmospheric eddy diffusion fluxes are set to zero in order to prevent the supply of angular momentum and heat from the surface through the sub-grid vertically diffusive process. Thus, the angular momentum and heat from the surface is supplied by the surface drag in the lowermost layer. 